\title{
Radiological Imaging in Transdiaphragmatic Extension of Hepatic Hydatid Cyst-A Case Report
}

\author{
Pramod Setty $\mathbf{J}^{1}$, Rushikesh Pokale ${ }^{2}$, Karuna Belide ${ }^{3}$, Ashwani Goyat ${ }^{4}$ \\ ${ }^{1}$ Professor and Head, Department of Radio-diagnosis, JJM Medical College, Davangere, Karnataka - 577 004, India \\ ${ }^{2,3,4}$ Post-Graduates, Department of Radio-diagnosis, JJM Medical College, Davangere, Karnataka - 577 004, India
}

\begin{abstract}
Thoracic extension of hepatic hydatid disease through transdiaphragmatic route occurs as rare but serious complication. We report a case presented as pleural effusion with consolidation on $X-R A Y$ and diagnosis of transdiaphragmatic rupture of hepatic hydatid was suspected on USG abdomen and confirmed by CT. Transdiaphragmatic thoracic involvement is one of the important to recognize as it ranges from simple to life-threatening complications. Diaphragmatic tear will be suspected on ultrasonography but CT is most important in showing defect. Transdiaphragmatic extension of hepatic hydatid is important to diagnose due to serious consequences like infection or destruction of surrounding structures. Radiological imaging in hepatic hydatid plays major role in diagnosing complications and planning management.
\end{abstract}

Keywords: Transdiaphragmatic extension, hepatic hydatid, complications.

\section{Introduction}

Hydatid disease is parasitic infection caused by larvae of Echinococcus. Echinococcusgranulosus is most common cause of human disease and less commonly by Echinococcus multilocularis ${ }^{1}$. Liver is most common organ involved in the human hydatid disease followed by lung ${ }^{2}$. Typical findings of hydatid disease are described, but findings because of its complications are less commonly described in the literature. At times, Echinococcal cyst rupture may be the first sign of hydatid disease in patients and it has infective or allergic complications ${ }^{3}$. Rarely invasion into thoracic cavity of the hepatic hydatid disease occurs through trans-diaphragmatic route as a serious complication ${ }^{4}$. Here, we report a case which presented primarily with respiratory symptoms and diagnosis of trans-diaphragmatic rupture was suspected on USG abdomen and confirmed by CT.

\section{Case Report}

A 45year old male farmer presented to the medicine OPD of CG Hospital, Davangere with complaints of chest pain, cough and fever since 3 days, breathlessness since 2 days. No history of expectoration orhaemoptysis.

On examination, respiratory sounds were decreased on the right upper and mid part, absent in the lower part in right hemithorax. Tenderness was noted in the right hypochondrium.

Patient was referred for chest X-Rayto the Department of radio-diagnosis. PA and lateral views of chest were obtained. On chest X-RayPA view, non-homogenous opacification noted in the right lung in the upper and midzone with round, well defined radiolucent lesion in the midzone suggestive of consolidation/collapse with likely infected lesion in mid-zone. Right CP angle, right hemidiaphragm and right border of heart are silhouetted due to homogenous opacification in the right lower zone suggestive of pleural effusion. Mild shift of mediastinum present towards right. On lateral view, curvilinear calcification was noted in the sub-diaphragmatic region. Patient was sent for USG Thorax and Abdomen.

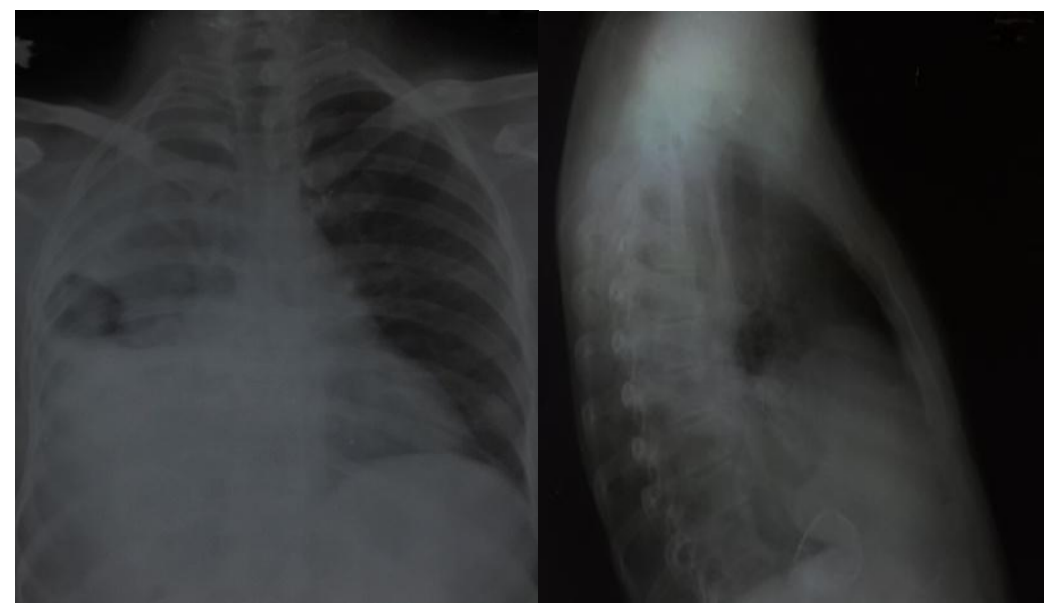

Figure 1: Chest radiograph (PA and lateral views) shows collapse consolidation involving right lung with infected cystic lesion in right mid lobe and right sided pleural effusion. Also ring like calcified lesion seen in the right subdiaphragmatic region. 


\section{International Journal of Science and Research (IJSR) \\ ISSN (Online): 2319-7064}

Index Copernicus Value (2013): 6.14 | Impact Factor (2014): 5.611

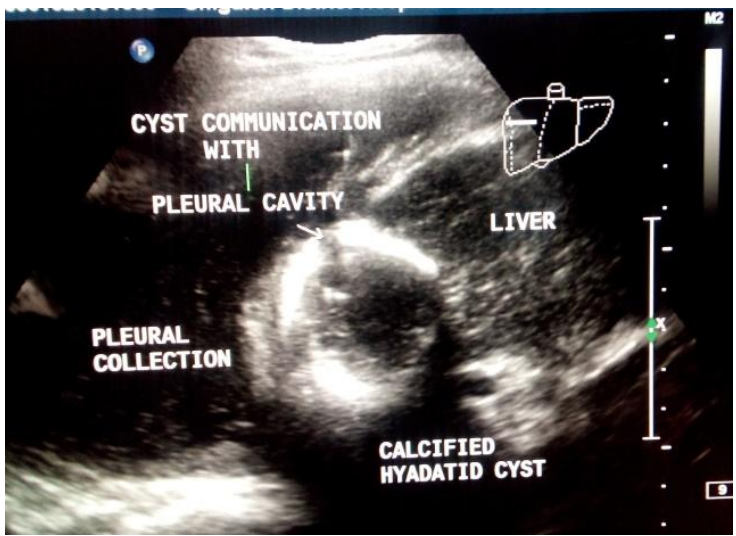

Figure 2: Gray scale ultrasound image showing incomplete rim calcified lesion in the immediate subdiaphragmatic region of liver and pleural effusion.

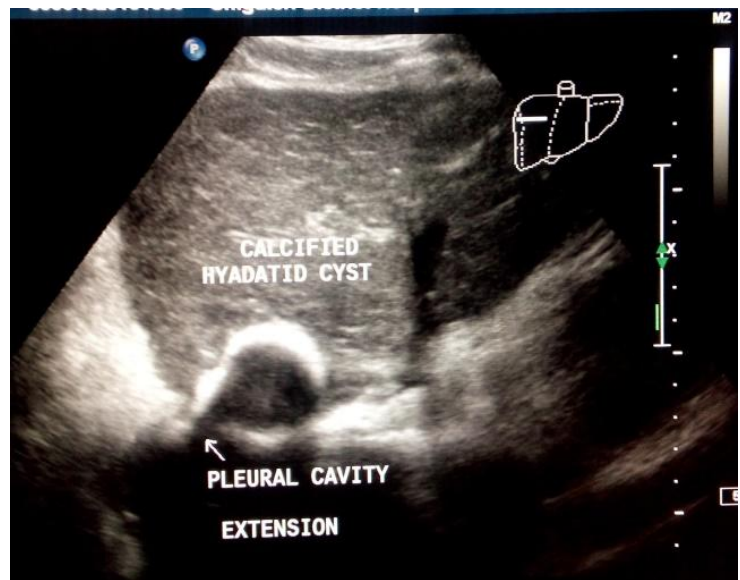

Figure 3: Subcostal scan through right upper quadrant showing blurred contour of diaphragm near the rim calcified lesion and pleural effusion

On ultrasound, massive pleural effusion was noted on the right side with underlying lung collapse. A round, welldefined hypoechoic lesion with peripheral calcification measuring $4.5 \times 3.5 \times 5 \mathrm{~cm}$ noted in the segment VII of liver. The peripheral calcification was not continuous near the diaphragm and diaphragmatic contour was blurred adjacent to it. So communication between cyst and pleural cavity is suspected. Another calcified lesion measuring 1.5 $\mathrm{X} 1 \mathrm{~cm}$ noted in the liver just below the first lesion. Spleen and bilateral kidneys were normal sonologically. There was no free fluid in the peritoneal cavity. Bowel appeared normal sonologically. Due to high suspicion of communication between cyst and pleural effusion, patient was referred for CECT-Thorax.
CECT of thorax and part ofupper abdomen was done; sagittal and coronal reconstructions were obtained. On axial images, well-defined, hypodense cystic lesion with peripheral wall calcification, measuring $4.5 \times 3.8 \times 5.2 \mathrm{cms}$ noted in the segment VII of liver. The calcified wall is not complete with breech in its superior aspect from which cyst is communicating with right pleural cavity through right hemidiaphragm and was confirmed on coronal and sagittal images. Another calcified focus immediately below the above mentioned lesion measuring $1.8 \mathrm{X} 1 \mathrm{X} 1 \mathrm{~cm}$ noted in segment VI of liver. Well defined thick walled cystic lesion measuring $7 \times 4.7 \times 3 \mathrm{~cm}$ noted in the right middle lobe of lung with few air pockets within it suggestive of infective etiology. Also massive right sided pleural effusion was noted with calcifications in the postero-inferior part of pleura on right side. Underlying collapse of part of right mid and lower zone was observed.

On lab investigations, Hb-10g\%, TLC- 16800 cells/cumm, Differential counts:neutrophils-81\%, eosinophils-9\%. ESR $58 \mathrm{~cm} / 1$ hour

Patient was advised indirect haemagglutination for microbiological confirmation but patient got discharged against medical advice.

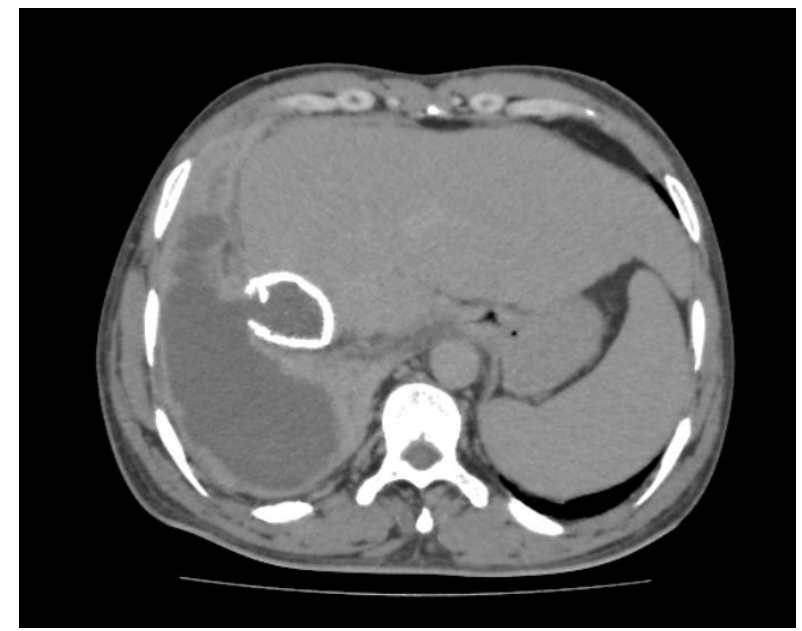

Figure 4: Axial CT image through upper abdomen showing communication between the incomplete calcified hepatic cystic lesion and right pleural collection through clearcut rent in the diaphragm. 


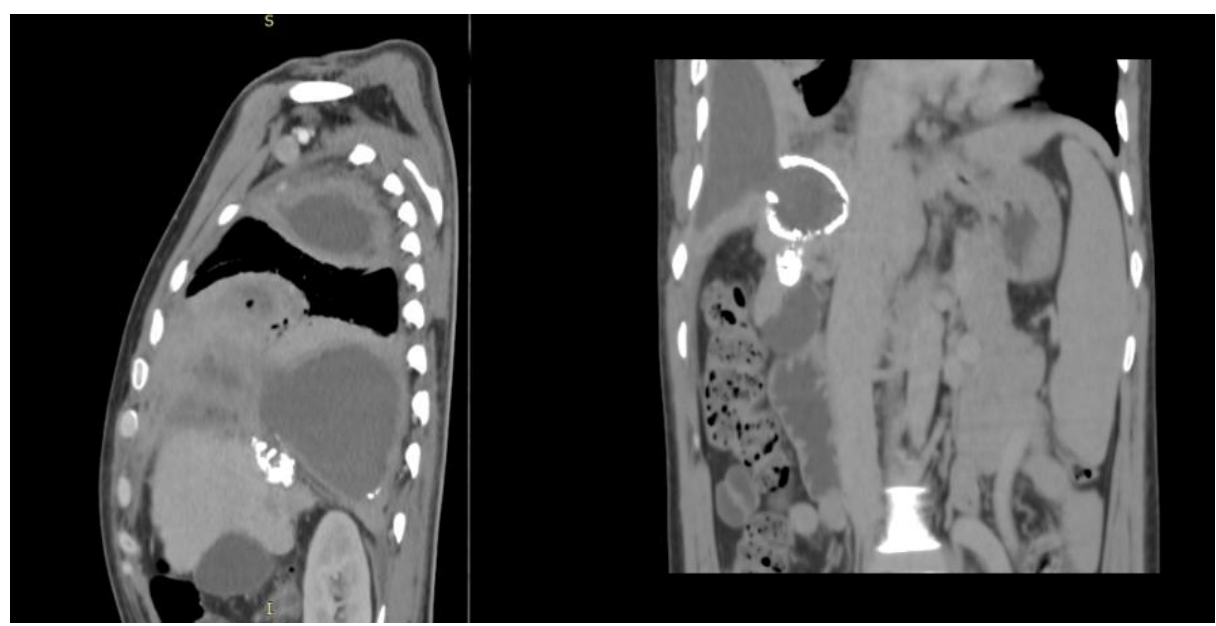

Figure 5: Sagittal and coronal reconstruction images confirming the cyst communication with pleural collection. Also sagittal image showing pleural calcification in the posteroinferior aspect and coronal image showing another calcified lesion just below rim calcified cystic lesion.

\section{Discussion}

Most common organ involved in the human hydatid disease is liver(75\%), followed by lung (15\%) and less commonly other locations $(10 \%)^{5}$. But simultaneous involvement of liver and lung is rare and in such cases right hemi-thorax is most commonly involved. Most common cause of liver cyst all over world is hydatid disease with right lobe being most common location ${ }^{6}$.

Calcified hydatid is seen in $20-30 \%$ of total hydatid cases and pattern of calcification is usually ring-like ${ }^{7}$. Calcification of pericyst is not the criteria for death of parasite but is indicated by complete calcification ${ }^{\mathbf{5}}$.

Complications of hydatid disease are difficult to diagnose as in many cases primary diagnosis of hydatid disease will not be there and patient presents at first with complications only. There are many complications of hepatic hydatid like cyst rupture, infection, communication with biliary tree, peritoneal seeding, and trans-diaphragmatic thoracic involvement. Among these complications, transdiaphragmatic thoracic involvement is one of the important to recognize as it ranges from simple to life-threatening complications.Main predisposing factors for cyst perforation are young age and superficial location.

Among hepatic hydatid cysts, $0.6-16 \%$ cases involve the thoracic cavity through trans-diaphragmatic route ${ }^{8}$.Thoracic involvement presents as either communication with pleural cavity or bronchial tree. Hepatic hydatid extending into pleural cavity is usually associated with secondary infection.

Diagnosis is made by ultrasonography and CT scan. Diaphragmatic tear will not be seen accurately in many cases but there may be blurring of diaphragmatic contour on ultrasonography. CT may show defect in the diaphragm on axial sections and confirmed mainly on sagittal and coronal reconstruction?. CT scan is the most important investigation for hepatic hydatid cyst as it gives most information regarding position and extent of hydatid cyst.

\section{Conclusion}

Trans-diaphragmatic rupture of hepatic hydatid is rare and must be included in the differential diagnosis of acute respiratory symptoms in the background of upper abdominal tenderness in endemic areas.

Radiological imaging in hepatic hydatid plays major role in diagnosing complications and planning management. As in case of transdiaphragmactic extension CT gives clear idea regarding the diaphragmatic rent and extent of thoracic involvement.

Surgery is the mainstay of treatment for such cases along with supportive post-op chemotherapy. Treatment of pulmonary hydatid is usually conservative with excision of cyst and maximum preservation of lung parenchyma. In case of trans-diaphragmatic thoracic extension of hepatic hydatid, radical surgery may be necessary due to serious consequences like infection or destruction of surrounding structures.

Due to absence of surgical confirmation, it was deduced that hydatid cyst was primarily hepatic causing intra-thoracic extension through trans-diaphragmatic route.

\section{References}

[1] Derici H, Tansug T, Reyhan E, Bozdag AD, Nazli O. Acute intraperitoneal rupture of hydatid cysts. World J Surg 2006; 30:1879-83.

[2] Barnes SA, Lillemoe KD. Liver abscess and hydatid cyst disease. In Maingot's abdominal operations.10th edition. 1997:1513-45.

[3] David B. Lewall, Scott J. McCorkell. "Rupture of Echinococcal Cysts: Diagnosis, Classification, and Clinical Implications.” AJR, 1986:391-394

[4] Flexinet JL, Mesters C, Cugat E. Hepatothoracic transdiaphragmatic echinococcosis. Ann Thoracic Surg 1988; 45 : 426-29.

[5] Pedrosa I, Saíz A, Arrazola J, Ferreirós J, Pedrosa C. Hydatid Disease: Radiologic and Pathologic Features 


\section{International Journal of Science and Research (IJSR) \\ ISSN (Online): 2319-7064}

Index Copernicus Value (2013): 6.14 | Impact Factor (2014): 5.611

and Complications1. RadioGraphics. 2000;20(3):795817.

[6] Ray S, Das K. Spontaneous intraperitoneal rupture of hepatic hydatid cyst with biliary peritonitis: a case report. Cases Journal . 2009; 2: 6511.

[7] Beggs I. The radiology of hydatid disease. AJR Am J Roentgenol 1985; 145:639-648.

[8] Gomez R, Moreno E, Leinaz C, et al. Diaphragmatic or transdiaphragmatic thoracic involvement in hepatic hydatid disease : Surgical trends and classification. World J Surg 1995;19: 714-19.

[9] Von Sinner WN. New diagnostic signs in hydatid disease, radiography, ultrasound, CT and MRI correlated to pathology. Eur J Radiol 1991; 12 : 150-59. 